\title{
Real-Time Tomography with Interactive 3D Visualization using tomviz
}

Robert Hovden ${ }^{1,2}$, Jonathan Schwartz ${ }^{1}$, Chris Harris ${ }^{3}$, Cory Quammen ${ }^{3}$, Shawn Waldon ${ }^{3}$, Peter Ercius ${ }^{4}$, Marcus D. Hanwell ${ }^{3}$, Yi Jiang ${ }^{5,6}$

1. Department of Materials Science and Engineering, University of Michigan, Ann Arbor, MI, USA

2. Applied Physics Program, University of Michigan, Ann Arbor, MI, USA

3. Kitware, Inc., Clifton Park, NY, USA

4. National Center for Electron Microscopy, Lawrence Berkeley National Laboratory, Berkeley, CA, USA

5. Department of Physics, Cornell University, Ithaca, NY, USA

6. X-ray Science Division, Advanced Photon Source, Argonne National Laboratory, Argonne, IL, USA.

Here we demonstrate interactive 3D visualization displayed in real time as tomographic reconstructions proceed and as new data arrives using the open-source tool, tomviz, publically available for download at www.tomviz.org .

Three-dimensional (3D) characterization at the nano- and meso-scale using scanning / transmission electron microscope (S/TEM) is now possible [1,2] but high-throughput tomography still requires innovative tools for reconstruction and visualization. Currently, the best tomographic reconstructions are obtained from algorithms that are slow and iterative and will run from hours to days depending upon the size of the data set and the algorithm(s) employed. Thus, it has been a longstanding desire to see a reconstruction and begin 3D analysis before it completes. Continuous feedback provides high-throughput and early diagnoses for 3D structure or opportunity to optimize experimental parameters for maximal reconstruction quality.

Using tomviz, a cross-platform tool, we demonstrate real-time visualization of electron tomography reconstructions as they proceed. Figure 1 shows screen shots taken from a live weighted back projection reconstruction visualized in tomviz - time proceeds from left to right. In the actual software, the 3D visualizations are dynamically updated throughout the computation. This means that scientists need not wait for a reconstruction to complete, or all data to be collected before beginning the interpretation of results. The iterative nature of tomographic methods allows tomviz to show intermediate results with minimal impact on performance. This enables interactive 3D analysis of the current reconstruction state while the reconstruction proceeds on a separate thread. A robust graphical interface allows objects to be rendered as shaded contours or volumetric projections (Fig. 2) and these objects can be rotated, cropped, or sliced [3]. Experimental nanomaterial datasets were used to validate live reconstruction [4].

Additionally, tomviz visualizes new data as it arrives. For aligned projections are provided from each new specimen tilt, the tomographic reconstruction quality improves in real-time. tomviz accomplishes this by monitoring data / directories for change, and upon arrival of new data, the data is imported and all associated 3D data visualizations are dynamically updated. If data processing routines are present in the pipeline - such as alignment, preprocessing, and reconstruction-all steps will automatically rerun. This capability opens radically new possibilities for developing high-throughput, real-time tomographic reconstruction algorithms. Ultimately, interactive real-time visualization will allow researchers to make early judgments to best answer scientific questions [5].

References:

[1] D. De Rosier, A. Klug, A. Nature 217 (1968), p. 130.

[2] P.A. Midgley, et al, Chemical Communications, 10 (2001), p. 907. 
[3] B. D.A. Leven et al, Microscopy Today 1 (2018), p. 12.

[4] E. Padgett et al, Microscopy and Microanalysis 23 (2017), p. 1150.

[5] tomviz is supported from DOE Office of Science contract DE-SC0011385.

Weighted Back Projection: Live Reconstructions

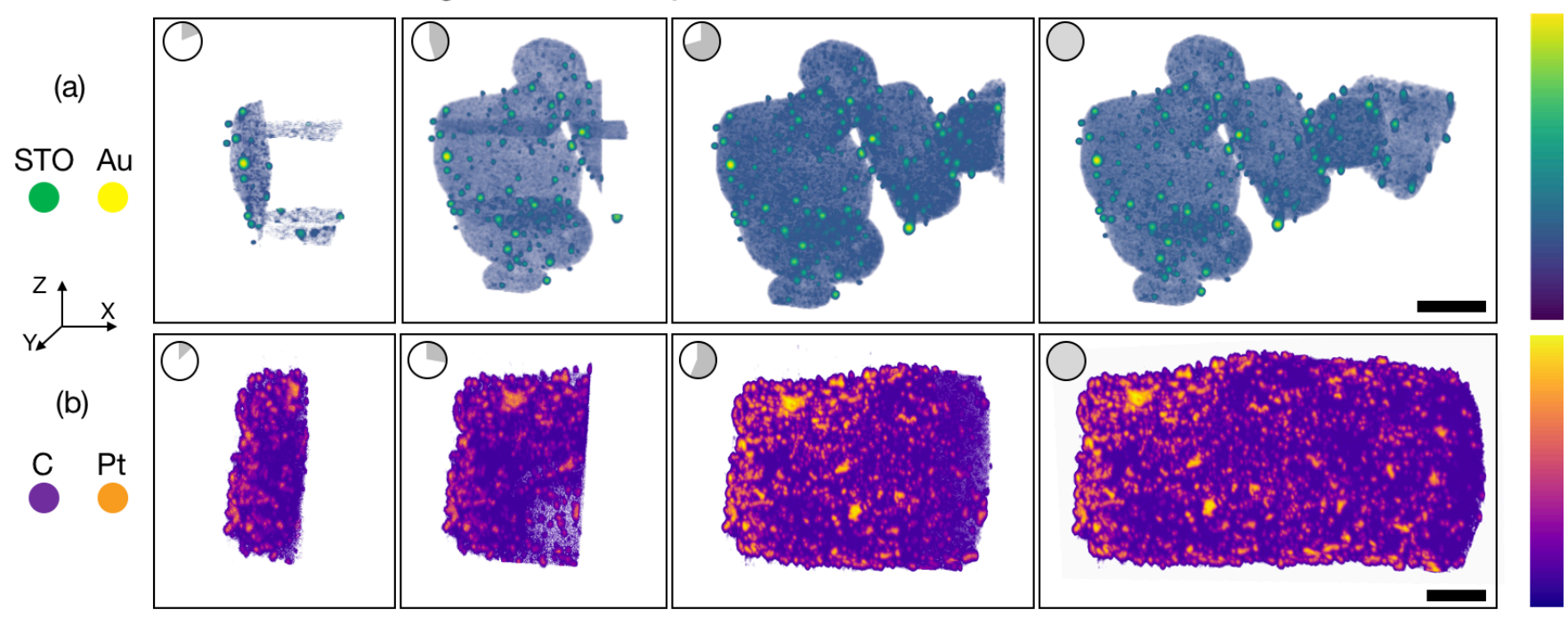

Figure 1. Live tomographic reconstruction in tomviz shown through freeze frames during progression of a weighted back projection algorithm (left to right). This unique capability allows users to interact and analyze the 3D structure throughout reconstruction. In the actual software the reconstruction updates in real time. (a) Live volume rendering of $\mathrm{Au} /$ strontium titanate (STO) nanoparticles. (b) Live volume rendering of platinum $(\mathrm{Pt})$ nanoparticles on a carbon support. Scale bar, $50 \mathrm{~nm}$.

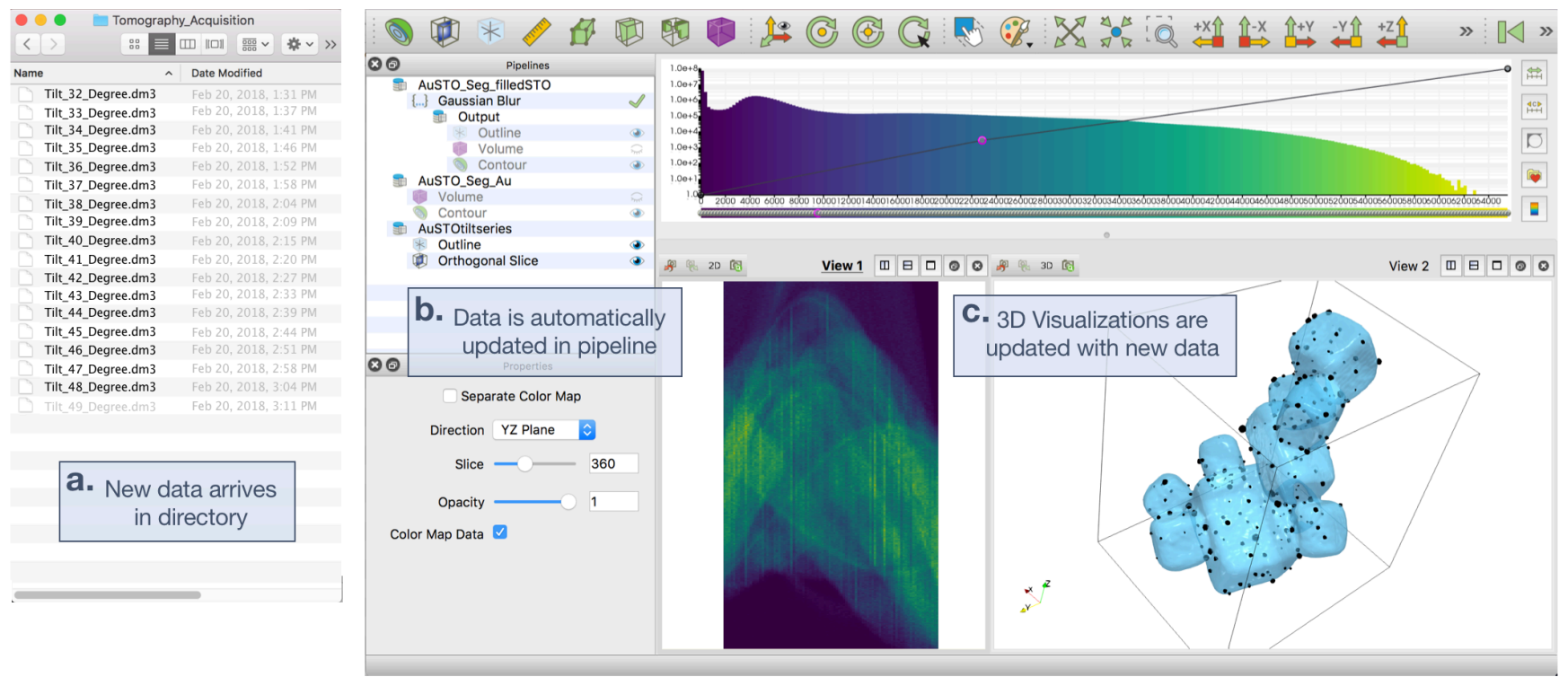

Figure 2. The tomviz graphical user interface monitors (a) tomography data within a directory and automatically reads new data into (b) the tomviz data pipeline and (c) updates 3D visualizations of the data in real time. Complex data pipelines can be constructed to automatically run when new data arrives. Interactive 3D visualizations, such as volumetric or contour surfaces, can be used in combination. (c) A sinogram and a corresponding reconstruction of Au/STO nanoparticles are visualized using tomviz. 